\title{
NATIONAL UNIVERSITY OF SINGAPORE
}

\section{Department Of Chinese Studies}



Applications are invited for teaching appointments in the Department of Chinese Studies from candidates who possess a relevant PhD degree, are able to teach in both Chinese and English, and have teaching/research interests in one of the following areas:

(1) Chinese Linguistics

(2) Chinese Philosophy (preferably Confucian studies)

(3) Translation

The Department is one of the largest departments in the Faculty of Arts \& Social Sciences, providing a multi-disciplinary approach to Chinese Studies, including areas such as linguistics, literature, history, philosophy, translation, tradition, culture and business. With a current strength of 40 staff, and more than 1000 students, the Department emphasises excellence in research and teaching. Funding for research is available and many staff have published in major international journals and academic presses.

Outstanding individuals with strong commitment to teaching and research are encouraged to send a curriculum vitae and three letters of recommendation to:

\author{
Assoc Prof Lee Cheuk Yin \\ Acting Head \\ Department of Chinese Studies \\ National University of Singapore \\ 10 Kent Ridge Crescent \\ Singapore 119260 \\ Tel: (65) 8743900 \\ Fax: (65) 7794167 \\ Email: chshead@nus.edu.sg
}

Visit our website at http://www.nus.sg/NUS info/Appoint/arts-chs.html for terms and conditions and application form.

Application Deadline: 31, March 1999

Only shortlisted candidates will be notified. 


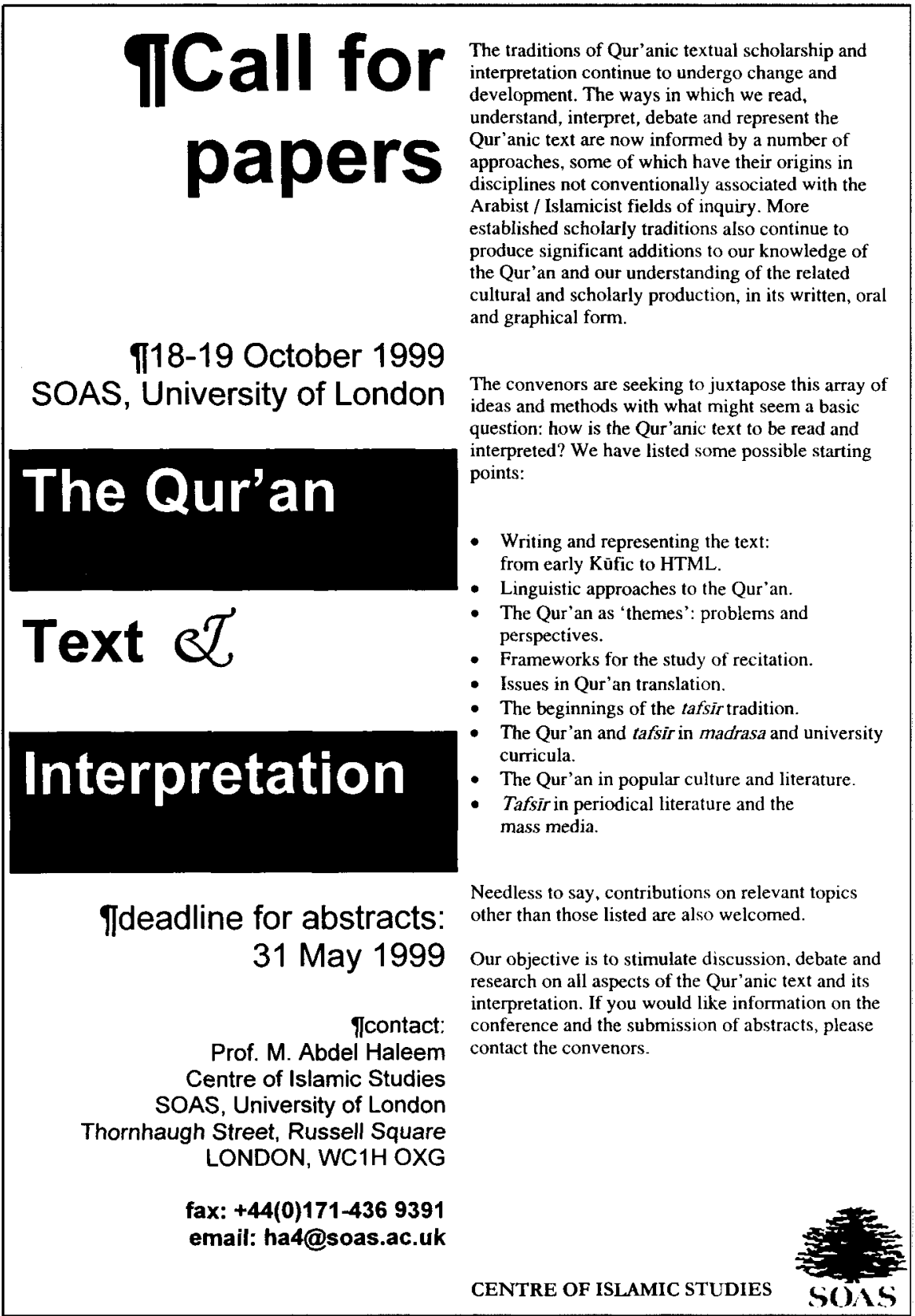

